\title{
Variational description of the Helium trimer using correlated hyperspherical harmonic basis functions
}

\author{
P. Barletta \\ Department of Physics and Astronomy, University College London, London WC1E 6BT, UK
}

A. Kievsky

Istituto Nazionale di Fisica Nucleare, Piazza Torricelli 2, 56100 Pisa, Italy

\begin{abstract}
A variational wave function constructed with correlated Hyperspherical Harmonic functions is used to describe the Helium trimer. This system is known to have a deep bound state. In addition, different potential models predict the existence of a shallow excited state that has been identified as an Efimov state. Using the Rayleigh-Ritz variational principle the energies and wave functions of both bound states have been obtained by solving a generalized eigenvalue problem. The introduction of a suitable correlation factor reduces considerably the dimension of the basis needed to accurately describe the structure of the system. The most recent helium-helium interactions have been investigated.
\end{abstract}

\section{INTRODUCTION}

The system He-He is greatly interesting both from a theoretical and an experimental point of view, and it has been object of intense investigations in the last years. Despite its simplicity, it is not easy to determine whether it supports or not a bound state. Experimentally, usual spectroscopy techniques are not suitable to its study, and only recently 佂目 diffraction experiments proved its existence, with a direct measurement of its bond length $\langle R\rangle$. Its binding energy has been estimated through the relation $\left|E_{b}\right| \approx \hbar^{2} / 4 m<R>^{2}$ and the $s$-wave scattering length has been estimated as $\left.\mathrm{a}_{0} \approx 2<R\right\rangle$. The most recent values for these quantities have been quoted in Ref. [4] after a new determination of the bond length by diffraction from a transmission grating. They are $\langle R\rangle=98 \pm 8$ a.u., $\left|E_{b}\right|=1.1+0.3 /-0.2 \mathrm{mK}$ and $\mathrm{a}_{0}=197+15 /-34$ a.u..

Theoretically difficulties in the description of the ${ }^{4} \mathrm{He}_{2}$ arise because the He-He interaction results from the subtraction of the huge energies of the separated atoms, which are only slightly different. Moreover, a $1 \%$ decrease of the strength of the interaction makes the system unbound. As a result of the continuous refinement in the past years of both experimental data and electronic structure computational techniques, several potential curves for He-He appeared in literature. Most of them are presented and compared in an article review by Janzen and Aziz [5]. However, due to the vivid interest in ${ }^{4} \mathrm{He}_{2}$, and to the difficulties in making a really accurate ab initio potential, newer and more accurate potentials have recently appeared in literature. Among the ones described in Ref. [5], the potentials called HFDB [6], LM2M2 [7], TTY [8] have been widely used in helium cluster calculations. Furthermore, two more up-to-date curves, namely SAPT1 and SAPT2 [9] are now available. The latter is believed to be the most accurate characterization of the He-He interaction yet proposed. These potentials are constructed on a completely ab initio calculations made by Korona et al. [10], using infinite order perturbation theory (SAPT) and very large orbital basis set. In addition, retarded dipole-dipole dispersion interaction is included over the range $0-100000$ a.u.(SAPT1), or the more appropriate 10-100000 a.u.(SAPT2).

All these five interactions support only a single bound state of zero total angular momentum. In Table I we summarize the different characteristics of each potential, as well as salient properties of the associated bound state. The latter has been computed solving the two-body Schrödinger equation by means of the Numerov algorithm . We used the value $\hbar^{2} / m=43.281307 \mathrm{~K}$ a.u. ${ }^{2}$. ¿From the table we can immediately see that the five potentials do not differ qualitatively among each other, though there is a spreading in the binding energy of the dimer $\left|E_{b}\right|$ of 0.51 $\mathrm{mK}$. The SAPT potentials predict the highest binding energies whereas the LM2M2 and TTY predictions are very close to each other and are the lowest ones. The differences observed in the binding energy are reflected in the mean values of the radius, $\langle R\rangle$ and $\left\langle R^{2}\right\rangle$, as well as in the scattering length $\mathrm{a}_{0}$. The estimate bond length $\langle R\rangle$ can be directly compared to the experiment and can be also used for an estimation of the binding energy $\left|E_{b}\right|$ and the scattering length $\mathrm{a}_{0}$ through the relations given in the first paragraph. Those values are shown in the last two rows of Table I and reasonably agree with the estimation of Ref. 何, in particular the results obtained with the LM2M2 and TTY potential are inside the quoted errors. We can also observe that the system is strongly correlated as its binding energy $\left|E_{b}\right|$ results from a large cancellation between the kinetic $\langle T\rangle$ and potential energy $\langle V\rangle$. Its spatial extension is considerably bigger than the range of the potential, as it is shown in Fig.1 where the LM2M2 potential and the dimer bound state wave function $\Phi_{d}$ are plotted. Finally, the scattering length $\mathrm{a}_{0}$ of the system is bigger 
than the range of the potential by an order of magnitude. All these features characterize the ${ }^{4} \mathrm{He}_{2}$ as the weakest, as well as the biggest, diatomic molecule found in nature so far. Moreover the bound state at practically zero energy suggests the possibility of observing an Efimov-like state in the triatomic compound [11, 12 ].

Along with the observations of small clusters of helium atoms, different theoretical methods have been used to study the properties of such systems. From the beginning it has been clear that standard techniques could have problems to describe those highly correlated structures and, accordingly, more sophisticated methods have been applied. In Ref. [13] the diffusion Monte Carlo (DMC) method was used to describe the ground state of He molecules up to 10 atoms. The ${ }^{4} \mathrm{He}_{3}$ has been extensively studied by different methods (see Ref. 114 and references therein). Theoretically it has been shown that the trimer has a deep ground state of about $126 \mathrm{mK}$ and a single $L=0$ excited state of about $2 \mathrm{mK}$. There are not bound states with $L>0$ [15. In Refs. 16, 17] the $L=0$ excited state has been studied, in particular looking at those characteristics identifying an Efimov state. In fact this state has the property of disappearing when the interaction strength is tuned with a parameter $\lambda$. For example, the excited state exists in the very narrow interval $0.97 \lesssim \lambda \lesssim 1.2$ for the LM2M2 potential. Though with slightly different values of $\lambda$, the same property holds for the other potentials mentioned above. Therefore the helium trimer gives the unique possibility of observing the Efimov effect, as the narrow range in $\lambda$ where the excited state ${ }^{4} \mathrm{He}_{3}^{*}$ appears contains the physical case $\lambda=1$.

In the present paper a set of correlated basis functions is used to describe the ${ }^{4} \mathrm{He}_{3}$ molecule. The correlated hyperspherical harmonic $(\mathrm{CHH})$ basis has been applied successfully in the ground state description of light nuclei [18]. Similarly to the cluster of helium, these systems are strongly correlated due to the high repulsion of the nucleonnucleon potential at short distances. Essentially the method consists in a decomposition of the wave function in terms of the hyperspherical harmonic $(\mathrm{HH})$ basis multiplied by a suitable correlation factor which takes into account the fact that the probability of finding any pair of atoms at distances smaller than 3 a.u. is practically zero. The correlation factor has been taken as product of one dimensional correlation functions $f(r)$ (Jastrow type). In Fig.1 $f(r)$ is compared to the dimer wave function, showing that both have the same short range behaviour.

The variational description of the trimer using the $\mathrm{CHH}$ basis is twofold. Firstly we would like to evaluate the capability of the correlated basis functions to describe a strongly correlated system. Special attention will be given to the convergence pattern of the energy for both the ground and excited states. In Ref. 14 calculated binding energies of the ground and excited states of the trimer obtained by different groups are given in correspondence with different interactions. The solutions of the Faddeev equations as well as variational methods and adiabatic approaches have been used in those calculations. For the very shallow excited state of the trimer, only few results using the variational method have been reported so far, showing the difficulty of describing this state with the required accuracy using such a technique. In the present work we will show that it is possible to obtain high precision upper bound estimates and wave functions for both the ground and excited states by solving a generalized eigenvalue problem. Moreover, a detailed study of the wave function will be performed. In particular the tail of the wave function will be analyzed with the extraction of the asymptotic constants. The second motivation of the present work regards the extension of the method to describe larger systems. In fact a complete study of the ground state and excited states of the tetramer has still to be performed. In this context the variational technique is promising and the present study should be considered a first step along this direction.

The paper is organized as follows. In the next section a discussion of the CHH basis for the systems of three atoms is given. The numerical results for the binding energy of the two bound states are given in Sect. 3. Some properties of the wave functions and the asymptotic constants are calculated in Sect. 4 whereas the main conclusions as well as some perspectives for the extension to larger systems are given in the last section.

\section{CHH BASIS}

In the present study the interaction between three helium atoms is taken as a sum of three pairwise potentials. The Hamiltonian of the system will be

$$
H=T+\sum_{i<j} V(i, j)
$$

where $T$ is the kinetic energy operator and $V(i, j)$ is the He-He interaction that in the present work will be taken as one of the potentials mentioned in the previous section.

Considering the helium atom as a spinless boson, the wave function for three identical spinless bosons can be written as a sum of three Faddeev-like amplitudes

$$
\Psi=\psi\left(\mathbf{x}_{1}, \mathbf{y}_{1}\right)+\psi\left(\mathbf{x}_{2}, \mathbf{y}_{2}\right)+\psi\left(\mathbf{x}_{3}, \mathbf{y}_{3}\right)
$$


where the sets of Jacobi coordinates $\left(\mathbf{x}_{i}, \mathbf{y}_{i}\right)(i, j, k=1,2,3$ cyclic) are:

$$
\begin{aligned}
& \mathbf{x}_{i}=\frac{1}{\sqrt{2}}\left(\mathbf{r}_{j}-\mathbf{r}_{k}\right) \\
& \mathbf{y}_{i}=\frac{1}{\sqrt{6}}\left(\mathbf{r}_{j}+\mathbf{r}_{k}-2 \mathbf{r}_{i}\right)
\end{aligned}
$$

Each $i$-amplitude has total angular momentum $L M$ and can be decomposed into channels

$$
\psi\left(\mathbf{x}_{i}, \mathbf{y}_{i}\right)=\sum_{\alpha} \Phi_{\alpha}\left(x_{i}, y_{i}\right)\left[Y_{\ell_{\alpha}}\left(\hat{x}_{i}\right) Y_{L_{\alpha}}\left(\hat{y}_{i}\right)\right]_{L M}
$$

A symmetric wave function requires $\ell_{\alpha}$ to be even. Moreover $\ell_{\alpha}+L_{\alpha}$ should be even for positive parity states.

Let us introduced the hyperspherical variables

$$
x_{i}=\rho \cos \phi_{i}, \quad y_{i}=\rho \sin \phi_{i}
$$

where $\rho$ is the hyperradius which is symmetric under any permutation of the three particles and $\phi_{i}$ is the hyperangle. In terms of the interparticle distances $r_{i j}=\left|\mathbf{r}_{i}-\mathbf{r}_{j}\right|$ the hyperradius reads:

$$
\rho=\frac{1}{\sqrt{3}} \sqrt{r_{12}^{2}+r_{23}^{2}+r_{31}^{2}} \text {. }
$$

Using the set of coordinates $\left[\rho, \Omega_{i}\right] \equiv\left[\rho, \phi_{i}, \hat{x}_{i}, \hat{y}_{i}\right]$, the volume element is $d V=\rho^{5} d \rho d \Omega_{i}=\rho^{5} d \rho \sin ^{2} \phi_{i} \cos ^{2} \phi_{i} d \phi_{i} d \hat{x}_{i} d \hat{y}_{i}$.

The two dimensional radial amplitude of eq.(4) is now expanded in terms of the CHH basis

$$
\Phi_{\alpha}\left(x_{i}, y_{i}\right)=\rho^{\ell_{\alpha}+L_{\alpha}} f\left(r_{12}\right) f\left(r_{23}\right) f\left(r_{31}\right)\left[\sum_{k} u_{k}^{\alpha}(\rho){ }^{(2)} P_{k}^{\ell_{\alpha}, L_{\alpha}}\left(\phi_{i}\right)\right] \text {, }
$$

where the hyperspherical polynomials are given by 19

$$
{ }^{(2)} P_{k}^{\ell_{\alpha}, L_{\alpha}}\left(\phi_{i}\right)=\mathcal{N}_{k}^{\ell_{\alpha}, L_{\alpha}}\left(\cos \phi_{i}\right)^{\ell_{\alpha}}\left(\sin \phi_{i}\right)^{L_{\alpha}} P_{k}^{l_{\alpha}+\frac{1}{2}, L_{\alpha}+\frac{1}{2}}\left(\cos 2 \phi_{i}\right)
$$

with $\mathcal{N}_{k}^{\ell_{\alpha}, L_{\alpha}}$ a normalization factor and $P_{k}^{a, b}$ a Jacobi polynomial. The quantum number $k$ is a non negative integer related to the grand orbital quantum number $K=\ell_{\alpha}+L_{\alpha}+2 k$. The product of the hyperspherical polynomial defined in eq.(8) times the spherical harmonics coupled to $L M$ in eq.(㺼 gives a standard three-body hyperspherical harmonic $(\mathrm{HH})$ function with defined total angular momentum.

The other ingredient in the expansion of eq.(4) is the correlation factor, taken in the present work of the product (Jastrow) type. Its role is to speed the convergence of the expansion describing those configurations in which two particles are close to each other. The use of Jastrow correlation factors has a long tradition in the description of infinite systems as nuclear matter or liquid helium [20] as well as in the description of light nuclei [18]. The wave function describing strongly interacting structures, in which the interaction is highly repulsive at short distances, is practically zero when the distance between any pair of particles is smaller than the repulsive core of the potential. The correlation factor imposes this behaviour as it can be seen from the specific form of the correlation function $f(r)$ given in Fig.1. The short range behaviour of $f(r)$ is governed by the two-body potential whereas its medium and long range form is not critical since the structure of the system will be constructed by the HH basis. A simple procedure to determine the correlation function for states in which the pair $(i, j)$ is in a relative state with zero angular momentum is to solve the following zero-energy Schrödinger-like equation 21]

$$
\left[-\frac{\hbar^{2}}{m}\left(\frac{\partial^{2}}{\partial r^{2}}+\frac{2}{r} \frac{\partial}{\partial r}\right)+V(r)+W(r)\right] f(r)=0
$$

where $V(r)$ is the He-He interaction used in the Hamiltonian of eq.(1). The additional term $W(r)$ is included to allow the function $f(r)$ to satisfy an appropriate healing condition. It is chosen as

$$
W(r)=W_{0} \exp (-r / \gamma)
$$

The specific value of $\gamma$ is not important provided that the ranges of the additional potential $W(r)$ and $V(r)$ are comparable 21]. Hereafter its value has been fixed to $\gamma=5$ a.u.. The depth $W_{0}$ is fixed requiring that $f(r) \rightarrow 1$ for values of $r$ greater than the range of the potential $V(r)$. 
The hyperradial functions $u_{k}^{\alpha}(\rho)$ of eq.(7) are taken as a product of a linear combination of Laguerre polynomials and an exponential tail:

$$
u_{k}^{\alpha}(\rho)=\sum_{m} A_{k, m}^{\alpha} L_{m}^{(5)}(z) \exp \left(-\frac{z}{2}\right)
$$

where $z=\beta \rho$ and $\beta$ is a non-linear variational parameter. Let $\mid \alpha, k, m>$ be a correlated completely symmetric element of the expansion basis, where $\alpha$ denotes the angular channels and $k, m$ are the indices of the hyperspherical and Laguerre polynomials, respectively. In terms of the basis elements the wave function (2) results

$$
\Psi=\sum_{\alpha, k, m} A_{k, m}^{\alpha} \mid \alpha, k, m>.
$$

The problem is to determine the linear coefficients $A_{k, m}^{\alpha}$. The wave function and energy of the different bound states are obtained by solving the following generalized eigenvalue problem

$$
\sum_{\alpha^{\prime}, k^{\prime}, m^{\prime}} A_{k^{\prime}, m^{\prime}}^{\alpha^{\prime}}<\alpha^{\prime}, k^{\prime}, m^{\prime}|H-E| \alpha, k, m>=0 .
$$

The dimension $N$ of the involved matrices is related to three indices: the number of angular channels $N_{\alpha}$, the number of hyperspherical polynomials per channel $K_{\alpha}$ and the number of Laguerre polynomials per channel $M_{\alpha}$. According to the Hylleraas-Undheim-MacDonald's theorem [22,23], there exists a one-to-one correspondence between the approximate energy levels $E_{i}(N)$ and the exact levels $\epsilon_{i} \equiv E_{i}(\infty)$, the $i$-th approximate level being an upper bound to the $i$-th exact level. Mathematically the following relations hold:

$$
E_{i+1}(N+1) \geq E_{i}(N) \geq E_{i}(N+1)
$$

and

$$
\lim _{N \rightarrow+\infty} E_{i}(N)=\epsilon_{i}
$$

The implementation of the method in the specific case of the helium trimer in which two bound states are known to exist, consists in solving the generalized eigenvalues problem for increasing values of $N$, until a convergence is achieved in the estimates of the ground state $E_{0}$ and excited state $E_{1}$. Moreover, an optimum choice of the non-liner parameter $\beta$ can be used to improve the pattern of convergence.

\section{BOUND STATE CALCULATIONS}

The generalized eigenvalue problem of eq.(13) can be solved to find bound states of general value of total angular momentum $L M$. Here we are interested in the ground and excited state of the helium trimer both having total angular momentum $L=0$. In such a case the angular dependence of each $i$-amplitude of the wave function reduces to a Legendre polynomial $P_{l}\left(\mu_{i}\right)$ with $\mu_{i}=\hat{x}_{i} \cdot \hat{y}_{i}$. Moreover, the angular channel with $\ell_{\alpha}=L_{\alpha}=0$ is, by far, the most important and it has been the first one to be considered. Contributions from successive channels, with $\ell_{\alpha}=L_{\alpha}>0$, are highly suppressed due to centrifugal barrier considerations and can be safely disregarded as it will be discussed latter.

The matrix elements defined in eq.(13) have been obtained numerically. In general, as the dimension of the matrices increases, numerical problems could arise from integrals containing polynomials of high degree. In fact, a high number of basis functions is expected in order to describe simultaneously both the ground and excited state, which have a completely different spatial extent. On the other hand, the correlation functions introduce a complicated structure at short distances. Therefore, a dense grid of integration points is necessary. The integrals have been performed in the set of coordinates $\left[\rho, \phi_{3}, \mu_{3}\right]$ using a Gauss formula in the variable $\mu_{3}$ and a Chebyshev Lobatto formula in the variable $\cos \left(2 \phi_{3}\right)$. Grids of 300 points for the first case and 3000 for the second have been used. In the variable $\rho$ the integrals have been performed on a scaled grid:

$$
\left\{\begin{array}{l}
\rho_{0}=h \\
\rho_{n}=\chi \rho_{n-1} \quad\left(n=1, n_{\max }\right)
\end{array}\right.
$$


with the choice $h=.07$ a.u., $\chi=1.008$, and $n_{\max } \approx 800$, covering the range $0-5000$ a.u.. A numerical accuracy of $10^{-3} \mathrm{mK}$ has been obtained in the calculation of the binding energies.

The convergence of the eigenvalues has been studied increasing the number of basis elements, restricting the discussion to one channel, namely the $\ell_{\alpha}=L_{\alpha}=0$ channel. In this case a totally symmetric wave function can be constructed for values of the quantum number $k=0,2, \ldots, k_{\max }$ (no symmetric function exists for $k=1$ ). Therefore, the number of hyperspherical polynomials $K_{0}$ included in a specific calculation is $k_{\max }$, except for $k_{\max }=0$ which corresponds $K_{0}=1$. The number of Laguerre polynomials is $M_{0}=m_{\max }+1$ with $m_{\max }$ the maximum degree considered. The total dimension of the problem to be solved is $N=K_{0} \cdot M_{0}$.

In Table II the convergence of $E_{0}$ and $E_{1}$ is shown as a function of $k_{\max }, m_{\max }$ for the LM2M2 potential. We observe that, while the ground state energy $E_{0}$ converges with a rather small basis set, for the excited state $E_{1}$ it is needed a much bigger basis (about one order of magnitude bigger). The ground state converged to the value $E_{0}=-126.36 \mathrm{mK}$ with $k_{\max }=20$ and $m_{\max }=20$ whereas the excited state converged to $E_{1}=-2.27 \mathrm{mK}$ with $k_{\max }=80$ and $m_{\max }=32$. In order to speed the convergence with respect to the Laguerre polynomials the value of the non linear parameter $\beta$ has been optimized. For the ground and excited state we have used $\beta=0.40 \mathrm{a} \cdot \mathrm{u}^{-1}$ and $\beta=0.10$ a.u. ${ }^{-1}$ respectively.

After the convergence of the first channel is achieved, the contribution of the channel $\ell_{\alpha}=L_{\alpha}=2$ can be evaluated. The first four linearly independent totally symmetric basis elements belonging to the second channel correspond to values of the grand angular quantum number $K=12,16,18,20$. The inclusion of these elements gives extremely small contributions to the binding energy of the two states and does not change the estimates given above. It is important to notice that the Jastrow correlation factor introduced contributions from channels with $\ell_{\alpha}=L_{\alpha} \geq 2$ already in a calculation limited to the first channel.

Special attention has been given to the study of the convergence with the non-linear parameter $\beta$. In Fig.2 we reported the ground and excited state energy curves as a function of $\beta$ for increasing values of $k_{\max }$. The number of Laguerre polynomials has been kept fixed at $m_{\max }=24$. For the ground state energy the upper curve corresponds to $k_{\max }=0$, i.e. only one hyperspherical polynomial has been taken into account, and the lower curve corresponds to $k_{\max }=20$. Results with larger values of $k_{\max }$ are not shown since they completely overlap with the result at $k_{\max }=20$. For the excited state energy the different curves correspond to $k_{\max }=20,40,60,80$. We observed that there is a region where the variation of $\beta$ does not appreciably affect the binding energies.

The variational method provides, in addition to an upper bound to the exact energy of the states, a variational estimate of the corresponding wave functions. Through the wave function it is possible to calculate a certain number of mean values characterizing the ground and excited state. In Tables III-IV, we computed the binding energy, the mean value of the kinetic energy, the potential energy, the interparticle distance $r_{i j}$, and the distance $r_{i}$ between the $i$-particle and the center of mass. The HFDB, LM2M2, TTY, SAPT1 and SAPT2 interactions have been considered. Other than typical observables, we also computed the asymptotic normalization constants $c_{\nu}$ for the two bound states, as defined in Refs. 24,25] and briefly described below, and the percentage $P_{d}^{\nu}$ of dimer-like structures in the trimer wave functions $\Psi_{\nu}(\nu=0,1)$.

The variational wave function is constructed in the present work as a sum of correlated products of polynomials with an exponential tail in the hyperradius. The short range behaviour of the wave function is governed by the correlation factor whereas in the medium and asymptotic region the expansion should reproduce the structure of the system. It is interesting to evaluate the flexibility of the correlated basis to reproduce correctly the asymptotic behaviour. Let us introduce the asymptotic function $\Theta_{\nu}(y)$ :

$$
\Theta_{\nu}(y)=\sqrt{2 q_{\nu}} \frac{e^{-q_{\nu} y}}{y}
$$

where $q_{\nu}=\sqrt{m\left|E_{\nu}-E_{d}\right| / \hbar^{2}}$, and $E_{\nu}$ and $E_{d}$ are respectively the trimer $(\nu=0,1)$ and the dimer binding energies. Here $y$ denotes the second Jacobi coordinate defined in eq.(33). In the configuration in which one atom is far from the other two the trimer w.f. for the ground and excited state $\Psi_{\nu}$ behaves asymptotically like:

$$
\Psi_{\nu} \stackrel{y \rightarrow \infty}{\longrightarrow} c_{\nu} \Theta_{\nu}(y) Y_{00}(\hat{y}) \Phi_{d}(\mathbf{x})
$$

where $\Phi_{d}$ is the dimer w.f., and $c_{\nu}$ the asymptotic constant. Therefore, the overlap function

$$
\mathcal{O}_{\nu}(y)=\int \Phi_{d}^{*}(\mathbf{x}) Y_{00}^{*}(\hat{y}) \Psi_{\nu} d \hat{y} d \mathbf{x}
$$

is proportional to $e^{-q_{\nu} y} / y$ as $y \rightarrow \infty$. In Fig.3 we plot the overlap functions $\mathcal{O}_{\nu}(y)$ and the asymptotic functions $\Theta_{\nu}(y)$ for both the ground and excited state. From the figure it is clear that the two curves approach to each other as 
the distance $y$ increases. We also observe the very large extension of the excited state. The asymptotic constants $c_{\nu}$ are obtained evaluating the ratio $\mathcal{O}_{\nu}(y) / \Theta_{\nu}(y)$ at large $y$ values and are given in Tables III and IV for the different interactions considered. For the ground state the five interactions produce similar values of $c_{0}$, though the values of LM2M2 and TTY are slightly smaller. Conversely, for the excited state the result obtained with SAPT2 is smaller than that obtained with the other potentials.

The percentage $P_{d}$ of a dimer-like structure inside the trimer is defined as:

$$
P_{d}^{\nu}=\int_{0}^{\infty}\left|\mathcal{O}_{\nu}(y)\right|^{2} y^{2} d y .
$$

The results for the ground and excited state are collected in Tables III and IV for the different interactions. For the trimer ground state the probability of a dimer-like structure is around 0.35 , whereas for the excited state this probability increases up to 0.75 for LM2L2 and TTY interactions. The two SAPT interactions predict a lower dimerlike structure, in particular SAPT2. This behaviour is related to the slightly tighter binding predicted by the SAPT potentials for the two bound states. As a general remark, the very high value of $P_{d}^{1}$ (nearly $70 \%$, compared to $35 \%$ of the ground state) suggests that the excited state of ${ }^{4} \mathrm{He}_{3}$ can effectively be pictured as a third particle orbiting around a two particle structure.

In Fig.4 we plotted some distribution functions relevant to understand the structure of the two bound states of the trimer. Namely, we plotted the pair distribution function $p\left(r_{i j}\right)$, which represents the probability to find the particles $i$ and $j$ at distance $r_{i j}$, and the mass distribution function $m\left(r_{i}\right)$, which is related to the probability to find the particle $i$ at distance $r_{i}$ from the center of mass of the system.

Our results for the ground state agree quite well with the ones published in literature $[16,17,26,28,27$. For the SAPT potentials we find that the bond becomes slightly tighter $(\approx 5 \%)$, as a result of the its more attractive well. But basically there are not qualitative differences in describing the trimer with any of the different pairwise interactions. There is a discrepancy in literature whether the main spatial arrangement of the three particles in the ground state is either a quasilinear or equilateral configuration [29]. Our results seem to agree with the latter. In fact, we can try to discriminate between the two by looking at the pair distribution and the mass distribution functions. As it is shown in Fig.4, the probability to find any particle in proximity of the CM is almost zero. This strongly suggests that the most probable configuration is the equilateral one. Moreover, the ratio between $\left\langle r_{i j}\right\rangle /\left\langle r_{i}\right\rangle$ is very close to the ideal $\sqrt{3}$ of equilateral triangle for all the potentials we used. Regarding the excited state, the presence of a two peaks structure in the pair distribution function agrees with the interpretation of such a state as composed by a two particle core surrounded by the third atom at a larger distance.

For the excited state the results do not depend qualitatively on the potential we use. This may look very surprising at a first glance, because this state is suspected to be an Efimov state, and consequently it is expected to be strongly affected by any minimum variation of the pairwise interaction. Following Refs. [16, 17, 27, , we studied the behaviour of ${ }^{4} \mathrm{He}_{3}^{*}$ as a function of the strength of the pairwise interaction. In Fig.5 we plotted the energy difference $E_{1}-E_{d}$ of the system as a function of the parameter $\lambda$ defined by:

$$
V_{H e-H e}=\lambda V_{L}
$$

where $V_{L}$ is the LM2M2 potential. We found that this state disappears both increasing and decreasing $\lambda$, in agreement with the claim that it is an Efimov state. Our results agree quite well with Refs. 16.17, where the peculiarity of such a behaviour has been widely discussed. To summarize it, we observed that the trimer begins to support an excited state at $\lambda \approx 0.975$; then, increasing $\lambda$, the binding energy firstly increases, until it achieves its maximum at $\lambda \approx 1.05$, and successively decreases, until it dissociates.

In order to compare the different pairwise interactions we assume that due to the very large extension of the w.f. compared to the range of the potential, the particles are not sensitive to the particular shape of it, but somehow to its average strength. Accordingly we define

$$
\lambda_{x}=\frac{\int_{\sigma_{x}}^{\infty} V_{x}}{\int_{\sigma_{L}}^{\infty} V_{L}},
$$

with $x=L, T, H, S 1, S 2$ in accordance with the LM2M2, TTY, HFDB, SAPT1 and SAPT2 interactions and $\sigma_{x}$ is the interparticle distance where the considered potential changes sign, i.e. $V_{x}\left(\sigma_{x}\right)=0$. In the smaller frame of Fig.5 we reported the different values of $\lambda_{x}$. It is worth to observe that the potentials do not differ so much to show dissimilar results, as all the points lie in a small interval of $\lambda_{L}=1$. In fact a plot in function of $\lambda$ for the other interactions shows that in all cases the physical case $\lambda=1$ is on the left of the minimum of the curve, as for the LM2M2 potential.

The most peculiar feature of an Efimov like system is that it disappears tightening the interaction among its components. Physically, such a behaviour could be explained by picturing the system like composed by a third 
particle orbiting around a two particle sub-system. Increasing the strength of the pairwise interaction makes the two particles tighter to each other, and the third one evaporates as a result of its very weak bound.

\section{CONCLUSIONS}

In the present work the helium trimer has been investigated using the most recent helium-helium potential models. The helium trimer wave function has been expanded in terms of the CHH basis. Then, the energies and wave functions of the ground and excited state have been obtained by solving a generalized eigenvalue problem. The Hylleraas-Undheim-MacDonald's theorem assures that the obtained results for the energy of the levels represent upper bounds to the exact values.

The strong repulsion of the He-He potential at short distances engenders some difficulties in the description of the three atoms system in terms of an expansion basis. Very large bases are then necessary in order to obtain a satisfactory description of the structure of both bound states. The structure is such that the probability of finding two atoms at short distances is close to zero and this type of behaviour is difficult to describe using, for example, a polynomial expansion. Correlation factors naturally introduce this behaviour accelerating the rate of convergence of the expansion basis. In particular the $\mathrm{CHH}$ basis combines a Jastrow correlation factor with the HH basis. The $\mathrm{CHH}$ basis has been used before in the description of nuclear systems [18] in order to take into account the strong repulsion of the nucleon-nucleon interaction at short distances. Here, the $\mathrm{CHH}$ basis has been used to study five different interactions in the description of the trimer. The pattern of convergence for the bound and excited state has been studied by increasing the number of basis elements. With a sufficient number of elements, the dependence on the non linear parameter $\beta$ is smooth. Therefore it is possible to obtain a simultaneous description of the bound states with high accuracy. The results are collected in Tables III-IV and are in close agreement with previous results obtained by different groups using the HFDB, LM2M2 and TTY interactions. The estimates for the binding energy are upper bounds to the exact levels and show that the variational method can be used to describe strongly correlated systems, as helium trimer, with results that are believed to be among the most accurate ones at present.

Some interesting aspects of the wave function have been studied. Its asymptotic behaviour in a configuration where one atom is moving away from the other two is given in eq.(18). In Fig.3 this behaviour is shown for the two bound states. From this study the asymptotic constants $c_{\nu}$ have been extracted. In some particular systems the asymptotic constants can be measured [30]. Moreover, the probability $P_{d}$ of a dimer-like structure inside the trimer has been calculated. This quantity gives a clear idea of the spatial structure of the molecule. For the ground state we obtained

$P_{d}^{0} \approx 0.35$ whereas for the excited state $P_{d}^{1} \approx 0.70$. This latter result suggests a configuration of two atoms in a dimer-like state with a third atom orbiting.

The present work should be consider as a first step in the use of the variational technique with correlated basis functions for describing small helium clusters. The extension of the method to study larger systems is feasible. The study of the bound states of the tetramer is at present underway and will be the subject of a forthcoming paper.

\section{ACKNOWLEDGMENTS}

The authors would like to acknowledge Prof. L. Bruch for helpful discussions.

[1] F. Luo, C. F. Giese and W. R. Gentry, J. Chem. Phys. 104, 1151 (1996)

[2] W. Schoellkopf and J. P. Toennies, J. Chem. Phys. 104, 1155 (1995)

[3] W. Schollkopf and J. P. Toennies, science 266, 1345 (1994)

[4] R. Grisenti, W. Schoellkopf, J. P. Toennies, G. C. Hegerfeldt, T. Kohler and M. Stoll, Phys. Rev. Lett. 85, 2284 (2000)

[5] A. R. Janzen and R. A. Aziz, J. Chem. Phys. 103, 9626 (1995)

[6] R. A. Aziz, F. R. W. McCourt and C. C. K. Wong, Molecular Physics 61, 1487 (1987)

[7] R. A. Aziz and M. J. Slaman, J. Chem. Phys. 94, 8047 (1991)

[8] K. T. Tang, J. P. Toennies and C. L. Yiu, Phys. Rev. Lett. 74, 1956 (1995)

[9] A. R. Janzen and R. A. Aziz, J. Chem. Phys. 107, 914 (1997)

[10] T. Korona, H. L. Williams, R. Bukowski, B. Jeziorski and K. Szalewicz, J. Chem. Phys. 106, 5109 (1997)

[11] V. Efimov, Phys. Lett. 33B, 563 (1970) 
[12] V. Efimov, Sov. J. Nucl. Phys. 12, 589 (1971)

[13] M. Lewerenz, J. Chem. Phys. 106, 4596 (1997)

[14] A. K. Motovilov, W. Sandhas, S. A. Sofianos, E. A. Kolganova, Eur. Phys. J. D 13, 33 (2001)

[15] L. W. Bruch, J. Chem. Phys. 110, 2410 (1999); T.G. Lee, B.D.Esry, B.C. Gou, and C.D. Lin, J. Phys. B34, L203 (2001)

[16] B. D. Esry, C. D. Lin and Chris H. Greene, Phys. Rev. A 54394 (1996)

[17] T. González-Lezana, J. Rubayo-Soneira, S.Miret-Artés, F. A. Gianturco, G. Delgado-Barrio and P. Villareal, Phys. Rev. Lett. 82, 1648 (1999)

[18] A. Kievsky, M. Viviani and S. Rosati, Nucl. Phys. A551, 241 (1993); M. Viviani, A. Kievsky and S. Rosati, Few-Body Syst. 18, 25 (1995)

[19] M. Fabre de la Ripelle, Annals of physics 123, 185 (1979)

[20] S. Fantoni and A. Fabrocini, in Microscopic Quantum Many-Body Theories and Their Applications, ed. by J. Navarro and A. Pols, Spingre-Verlag 1998, p. 119.

[21] S. Rosati, M. Viviani and A. Kievsky, Few-Body Syst. 9, 1 (1990); M. Viviani, A. Kievsky and S. Rosati, Nuovo Cim. 105A, 1473 (1992)

[22] E. A. Hylleraas and B. Undheim, Z. Phys 65, 759 (1930)

[23] J. K. L. MacDonald, Phys. Rev. 43, 830 (1933)

[24] J. L. Friar, B. F. Gibson, D. R. Lehman and G. L. Payne, Phys. Rev. C 25, 1616 (1982)

[25] H. Kameyama, M. Kamimura and Y. Fukushima, Phys. Rev. C 40, 974 (1989)

[26] E. Nielsen, D. V. Fedorov and A. S. Jensen, J. Phys. B 31, 4085 (1998)

[27] T. González-Lezana, J. Rubayo-Soneira, S.Miret-Artés, F. A. Gianturco, G. Delgado-Barrio and P. Villareal, J. Chem. Phys. 110, 9000 (1999)

[28] D. Blume, Chris H. Greene and B. D. Esry, J. Chem. Phys. 1132145 (2000)

[29] B.D. Esry, C.D. Lin, C.H. Greene, and D. Blume, Phys. Rev. Lett. 86, 4189 (2001); T. González-Lezana et al.,Phys. Rev. Lett. 86, $4190(2001)$

[30] B. Kozlowska, Z. Ayer, R. K. Das, H. J. Karwowski and E. J. Ludwig, Phys. Rev. C50, 2695 (1994) 


\begin{tabular}{|c|c|c|c|c|c|c|c|}
\hline & & HFDB & LM2M2 & TTY & SAPT1 & SAPT2 & Ref. \\
\hline$\epsilon$ & $(\mathrm{K})$ & 10.95 & 10.97 & 10.98 & 11.05 & 11.06 & \\
\hline$r_{m}$ & (a.u.) & 5.599 & 5.611 & 5.616 & 5.603 & 5.602 & \\
\hline$\sigma$ & (a.u.) & 4.983 & 4.992 & 5.000 & 4.987 & 4.987 & \\
\hline$\overline{\left|E_{b}\right|}$ & $(\mathrm{mK})$ & 1.685 & 1.303 & 1.313 & 1.733 & 1.816 & \\
\hline$\langle T\rangle$ & $(\mathrm{mK})$ & 112.2 & 99.43 & 99.93 & 115.0 & 117.8 & \\
\hline$-\langle V\rangle$ & $(\mathrm{mK})$ & 113.9 & 100.7 & 101.2 & 116.8 & 119.6 & \\
\hline $\mathrm{a}$ & (a.u.) & 170.5 & 191.4 & 190.7 & 166.9 & 163.2 & \\
\hline$\overline{\hbar^{2} / 4 m\langle R\rangle^{2}}$ & $(\mathrm{mK})$ & 1.403 & 1.127 & 1.136 & 1.462 & 1.525 & $1.1+0.3 /-0.2$ \\
\hline $2\langle R\rangle$ & (a.u.) & 175.6 & 195.9 & 195.2 & 172.1 & 168.5 & $197+15 /-34$ \\
\hline
\end{tabular}

TABLE I. Characteristic values of the different potentials and their relative bound states. $R$ represents the He-He distance, $\epsilon$ is the strength of the potential at its point of minimum $r_{m}$ and $\sigma$ is the distance at which the potential changes sign.

\begin{tabular}{|c|c|c|c|c|c|}
\hline & \multicolumn{5}{|c|}{ ground state } \\
\hline \multirow{2}{*}{$\begin{array}{l}k_{\max } \\
m_{\max }\end{array}$} & \multirow{2}{*}{$\begin{array}{c}5 \\
E_{0}(\mathrm{mK})\end{array}$} & \multirow{2}{*}{$\begin{array}{c}10 \\
E_{0}(\mathrm{mK})\end{array}$} & \multirow{2}{*}{$\begin{array}{c}15 \\
E_{0}(\mathrm{mK})\end{array}$} & \multicolumn{2}{|c|}{20} \\
\hline & & & & $E_{0}(\mathrm{mK})$ & $T_{0}(\mathrm{mK})$ \\
\hline 4 & -120.376 & -120.689 & -120.726 & -120.737 & 1769.481 \\
\hline 8 & -126.080 & -126.274 & -126.286 & -126.288 & 1662.829 \\
\hline 12 & -126.143 & -126.337 & -126.348 & -126.349 & 1660.232 \\
\hline 16 & -126.148 & -126.342 & -126.353 & -126.354 & 1660.185 \\
\hline 24 & \multicolumn{5}{|c|}{ excited state } \\
\hline$k_{\max }$ & 20 & 40 & 60 & \multicolumn{2}{|c|}{80} \\
\hline$m_{\max }$ & $E_{1}(\mathrm{mK})$ & $E_{1}(\mathrm{mK})$ & $E_{1}(\mathrm{mK})$ & $E_{1}(\mathrm{mK})$ & $T_{1}(\mathrm{mK})$ \\
\hline 8 & -1.168 & -1.579 & -1.612 & -1.622 & 139.535 \\
\hline 12 & -1.523 & -2.097 & -2.148 & -2.160 & 127.139 \\
\hline 16 & -1.555 & -2.150 & -2.222 & -2.237 & 123.526 \\
\hline 32 & -1.567 & -2.162 & -2.242 & -2.265 & 121.935 \\
\hline
\end{tabular}

TABLE II. Convergence of the LM2M2 ground state energy $E_{0}$ and excited state energy $E_{1}$ for increasing values of the order of hyperspherical polynomials $k_{\max }$ and Laguerre polynomials $m_{\max }$. In the last column the convergence for the kinetic energy is shown. Basis states with $\ell_{\alpha}=L_{\alpha}=0$ have been considered. The non linear parameter $\beta$ has been fixed to 0.40 a.u. ${ }^{-1}$ for the ground state and 0.10 a.u. ${ }^{-1}$ for the excited state. 


\begin{tabular}{|c|c|c|c|c|c|c|}
\hline & & HFDB & LM2M2 & TTY & SAPT1 & SAPT2 \\
\hline$B$ & $(\mathrm{mK})$ & 133.0 & 126.4 & 126.4 & 133.8 & 135.1 \\
\hline$<T>$ & $(\mathrm{mK})$ & 1698 & 1660 & 1662 & 1707 & 1715 \\
\hline$-<V>$ & $(\mathrm{mK})$ & 1831 & 1787 & 1788 & 1841 & 1850 \\
\hline$<r_{i}>$ & (a.u.) & 10.38 & 10.51 & 10.49 & 10.36 & 10.25 \\
\hline$\sqrt{<r_{i}^{2}>}$ & (a.u.) & 12.11 & 12.28 & 12.26 & 12.09 & 12.03 \\
\hline$<r_{i j}>$ & (a.u.) & 17.98 & 18.21 & 18.16 & 17.95 & 17.77 \\
\hline$P_{d}^{0}$ & & 0.3614 & 0.3310 & 0.3311 & 0.3619 & 0.3539 \\
\hline
\end{tabular}

TABLE III. Binding energy and mean values of the kinetic and potential energy of the helium trimer ground state calculated for different pairwise interactions. The mean values and square root mean values of the distance $r_{i}$ of particle $i$ from the CM, and the interparticle distance $r_{i j}$ are also given. In the last two rows the asymptotic constant $c_{0}$ and the probability of a dimer-like structure are reported.

\begin{tabular}{|c|c|c|c|c|c|c|}
\hline & & HFDB & LM2M2 & TTY & SAPT1 & $\overline{\mathrm{SAPT} 2}$ \\
\hline$B$ & $(\mathrm{mK})$ & 2.735 & 2.265 & 2.277 & 2.788 & 2.885 \\
\hline$<T>$ & $(\mathrm{mK})$ & 134.1 & 121.9 & 122.4 & 135.7 & 137.8 \\
\hline$-<V>$ & $(\mathrm{mK})$ & 136.8 & 124.2 & 124.7 & 138.5 & 141.7 \\
\hline$<r_{i}>$ & (a.u.) & 87.24 & 94.00 & 93.67 & 83.07 & 76.22 \\
\hline$\sqrt{\left.<r_{i}^{2}\right\rangle}$ & (a.u.) & 103.5 & 111.1 & 109.5 & 96.67 & 81.00 \\
\hline$<r_{i j}>$ & (a.u.) & 145.6 & 157.0 & 150.7 & 139.1 & 125.0 \\
\hline$\sqrt{\left\langle r_{i j}^{2}\right\rangle}$ & (a.u.) & 177.7 & 192.5 & 183.7 & 167.5 & 141.1 \\
\hline$c_{1}$ & & 1.20 & 1.24 & 1.24 & 1.19 & 1.09 \\
\hline$P_{d}^{1}$ & & 0.7266 & 0.7462 & 0.7461 & 0.7030 & 0.5816 \\
\hline
\end{tabular}

TABLE IV. Binding energy and mean values of the kinetic and potential energy of the helium trimer excited state calculated for different pairwise interactions. The mean values and square root mean values of the distance $r_{i}$ of particle $i$ from the CM, and the interparticle distance $r_{i j}$ are also given. In the last two rows the asymptotic constant $c_{0}$ and the probability of a dimer-like structure are reported.

Figure Captions

Figure 1. The LM2M2 potential $V$, its corresponding ground state wave function $\Phi_{d}$ as well as the correlation function $f$ as a function of the interparticle distance $r$.

Figure 2. The ground state energy $E_{0}$ and excited state energy $E_{1}$ as a function of the non linear parameter $\beta$. For the ground state the curves corresponding to $k_{\max }=0,20$ are shown, whereas for the excited state the curves are given in correspondence to $k_{\max }=20,40,60,80$. The number of Laguerre polynomials has been fixed to $m_{\max }=24$ in all cases.

Figure 3. Overlap functions $\mathcal{O}_{\nu}(y)$ (solid line) and asymptotic functions $\Theta_{\nu}(y)$ (dashed line) for the ground state $(\nu=0)$ and excited state $(\nu=1)$.

Figure 4. Distribution functions $p\left(r_{i j}\right)$ and $m\left(r_{i}\right)$ for the ground and excited state of the helium trimer.

Figure 5. The energy difference $E_{1}-E_{d}$ as a function of $\lambda$. In the small frame the positions in the curve of the different values for $\lambda_{x}$ calculated as explained in the text are given. 


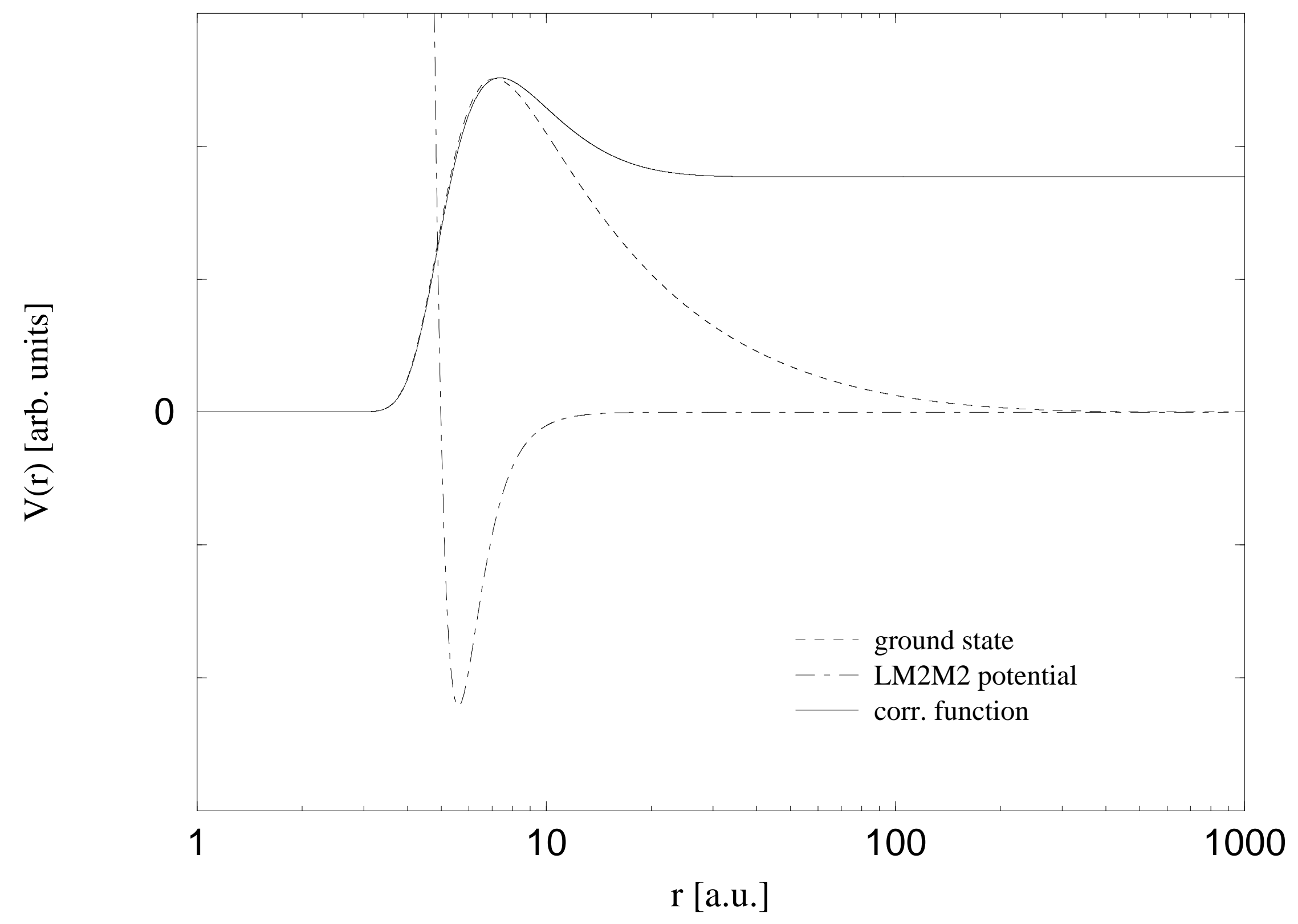



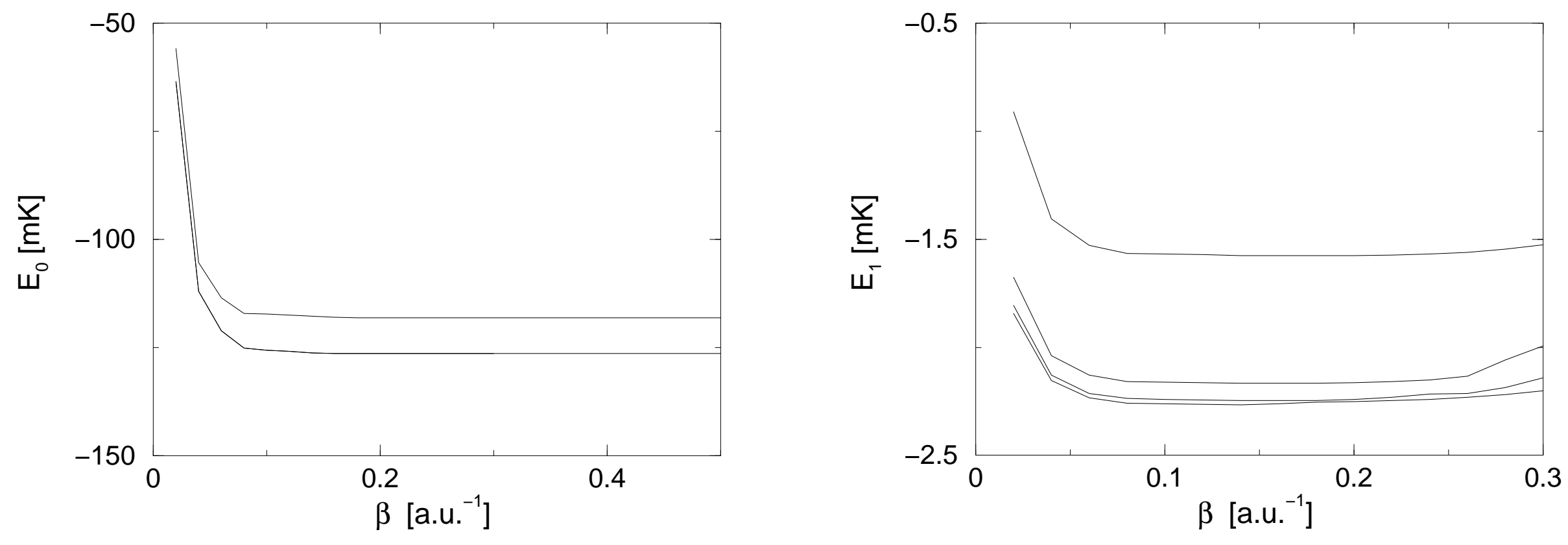

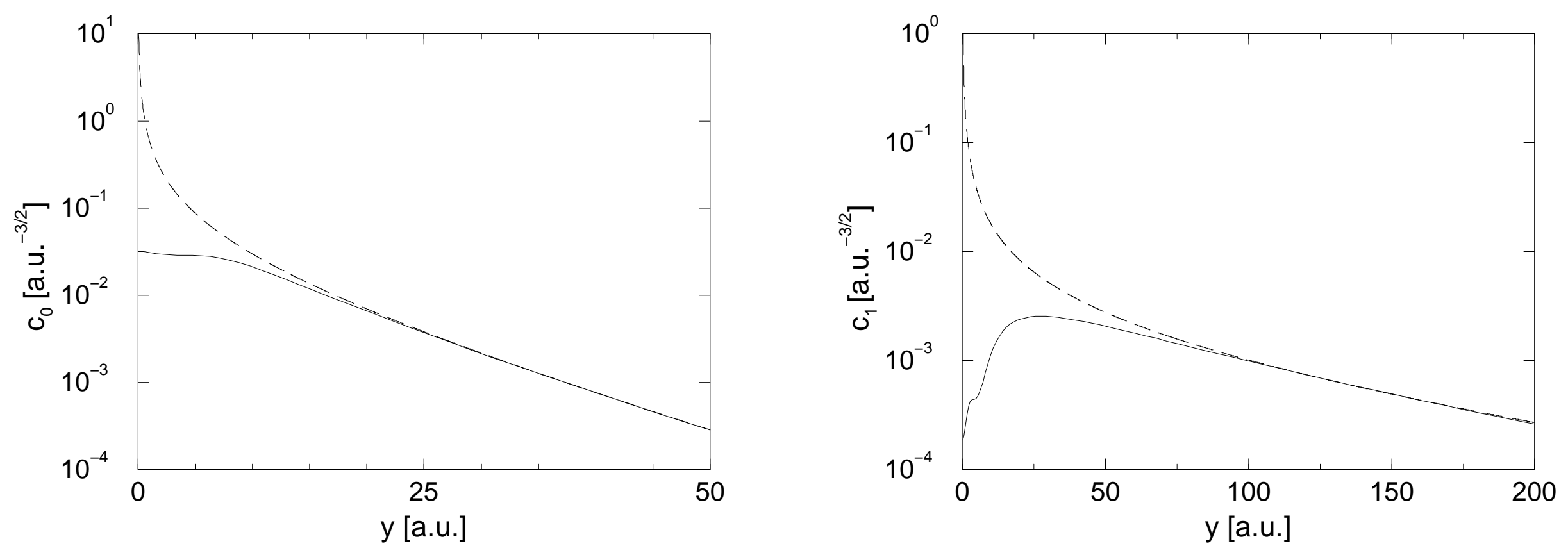

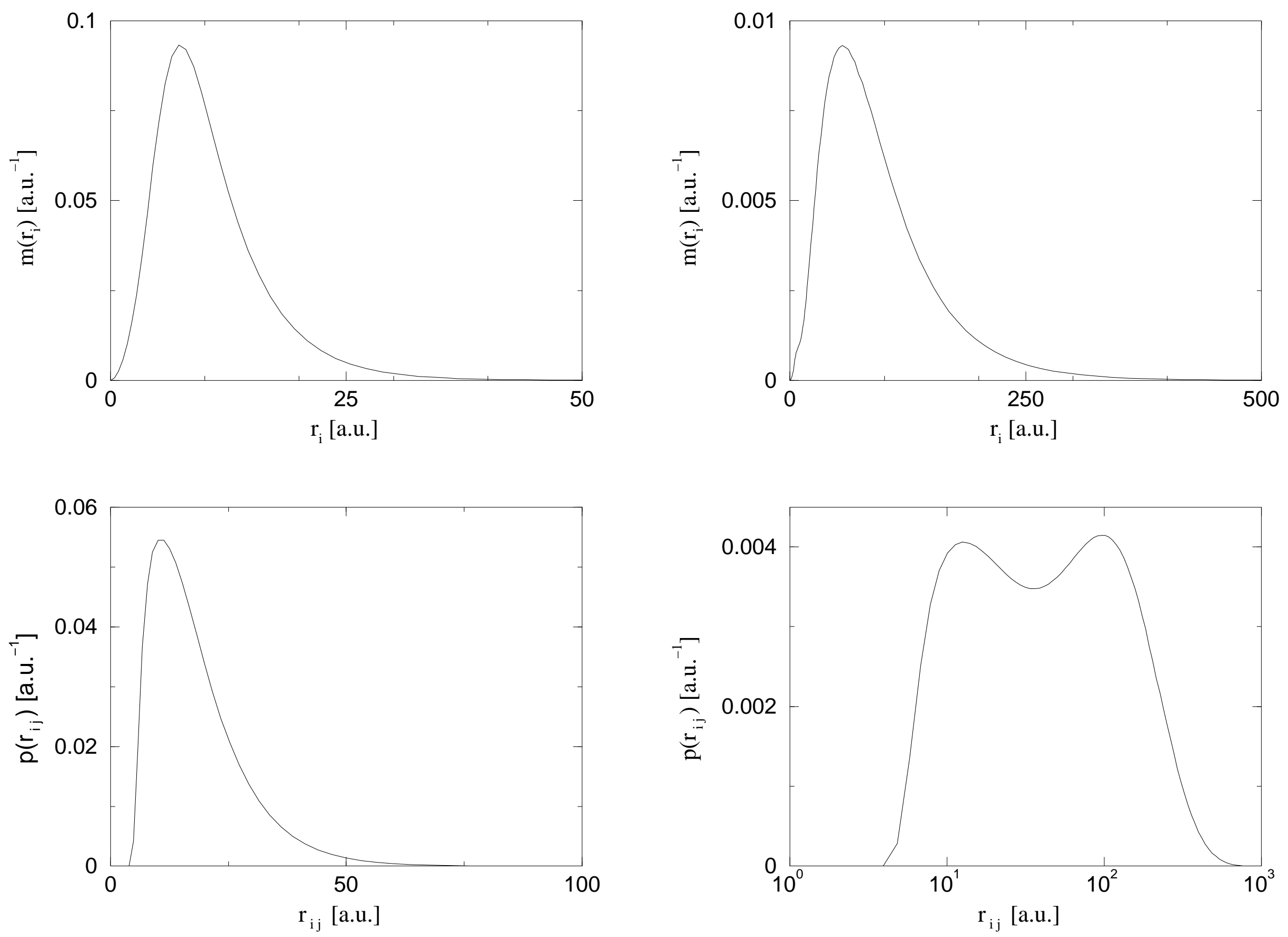


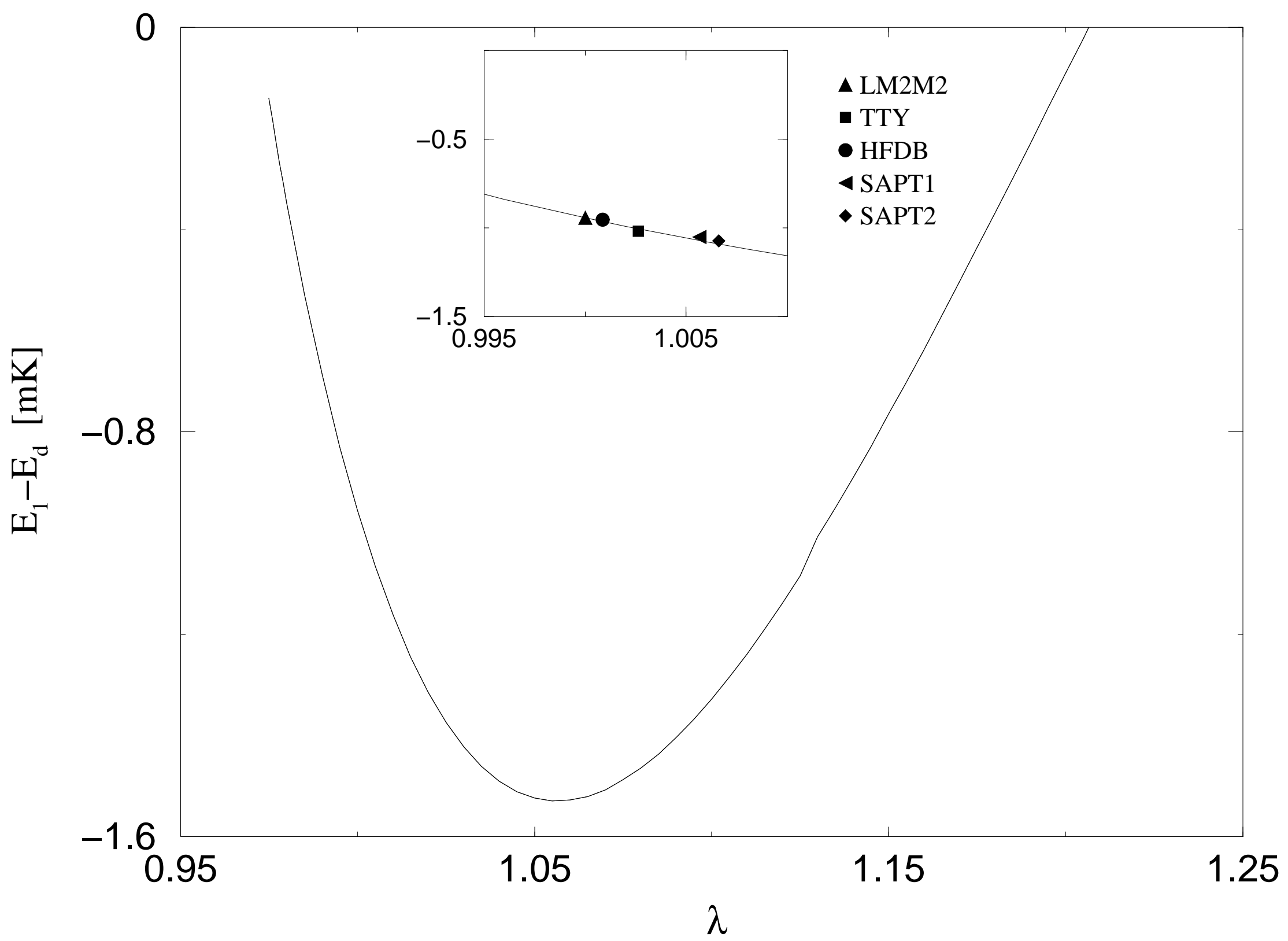

